Ashton Peters · J. Geoffrey Chase • Elijah E.W. Van Houten

\title{
Estimating elasticity in heterogeneous phantoms using Digital Image Elasto-Tomography
}

Received: date / Accepted: date

\begin{abstract}
Results from the application of a Digital Image Elasto-Tomography (DIET) system to elasticity distribution estimation in heterogeneous phantoms are presented. Two simple phantoms comprising distinct hard and soft regions were created from silicone, with harmonic surface motion data captured using a steady-state stereo imaging setup. A two-parameter approach to estimating stiffness distribution was used, applying both corroborative and contradictive methods to the inverse problem. The contradictive approach proved more robust in the presence of error in a priori stiffness assumption. These contrast based methods have the ability to reduce the number of parameters required for shape-based stiffness reconstructions, and present a novel approach to inclusion imaging in elastography.
\end{abstract}

\section{Introduction}

Elastography is a developing field focused on evaluating the elasticity distribution within tissue. While several implementations of elastographic imaging methods are currently being developed, the general format for the elastographic imaging process is very similar across all methods. This process involves first generating an image of the displacements that occur in the tissue given some external actuation, and then converting that displacement information into an image that relates to the stiffness distribution within the tissue. The clinical value of elastographic imaging information has been recently demonstrated [3], confirming evidence from mechanical testing results

A. Peters · J.G. Chase · E.E.W. Van Houten

Department of Mechanical Engineering, University of Canterbury, Christchurch, New Zealand

Tel.: $+64-3-3642987 \times 7486$

Fax: +64-3-3642078

E-mail: ashton.peters@student.canterbury.ac.nz 
that the contrast in elastic modulus between healthy and benign tissue could be of medical interest $[4,16]$.

Interest in imaging elasticity contrast has existed for some time. Initially conceived as an extension of ultrasound imaging $[7,8]$, elastographic methods were later developed using magnetic resonance based approaches $[6,5]$ and have been investigated using x-ray based methods [14]. Both ultrasound elastography (USE) and magnetic resonance elastography (MRE) have recently demonstrated success in clinical settings $[2,17,15]$, indicating that elasticity imaging may have a valuable role to play in the treatment of various medical conditions.

Recently, an elasticity imaging technique based on photographically generated surface motion data has been suggested [11,9]. Digital Image ElastoTomography (DIET) uses calibrated digital imaging arrays to capture the steady state vibration patterns across the surface of a specimen undergoing controlled actuation. These motions are then used to generate an estimate of the internal stiffness distribution of the specimen. The DIET system offers two points of contrast with USE and MRE: the digital imaging hardware used to drive the DIET process is much less expensive and infrastructurally intensive than the MR imaging equipment used in MRE; additionally, the 3D elastographic descriptions that result from the DIET process are much more amenable to co-registration across regular time intervals for screening as compared to USE, where the "freehand" nature of the imaging process and a lack of transverse imaging capability hinders screening potential. The DIET method therefore offers a potential means towards using elasticity contrast as a screening modality.

The reconstruction of internal elasticity distribution based on external motion measurements is a challenging problem [13]. The presence of local minima makes the direct application of traditional tomographic image reconstruction techniques ineffective. One way of improving the feasibility of this reconstruction problem is by representing the internal stiffness distribution with a parameterized shape based functional description. The work presented here describes an investigation into the application of the DIET technique to heterogeneous gelatin phantoms. We examine the use of corroborative and contradictive approaches to the test assumptions used in parameterized, shape based elasticity function estimation.

\section{Methods and Materials}

Two heterogeneous silicone phantoms of the same size order as a human breast were used for experimental motion capture. Estimates of the mechanical property distribution of the heterogeneous phantoms were made by comparing the surface motion measured in the laboratory with displacements calculated from a range of Finite Element (FE) simulations. 


\subsection{Phantom Displacement Imaging}

Two symmetric, cylindrical phantoms representing simple heterogeneous geometries were used for motion capture. Each phantom was $74 \mathrm{~mm}$ in diameter and $74 \mathrm{~mm}$ in height, and was made from a combination of soft and hard silicone gel. Stacked and concentric geometries were chosen for consistency with previous simulation studies [9]. These geometries provide a progression between a simple homogeneous case, and the expected geometry in a clinical scenario, where a stiff inclusion is completely contained within a softer body. The stacked phantom shown in Figure 1(a) comprised a hard silicone layer below a soft silicone layer, with a horizontal interface between the regions. The concentric phantom contained a cylindrical core of hard silicone set within soft silicone, and is shown in Figure 1(b). The heterogeneous phantoms were made using the same silicone manufacturing method, but at a different time, as homogeneous phantoms prepared for a separate study [10].

During the phantom manufacturing process, a small amount of flesh tone pigment was added to the hard silicone. The soft silicone was left unpigmented, resulting in a colorless clear solid. This color contrast was used to aid manufacturing and to clearly indicate the interface position between the hard and soft silicone regions. A column of fifteen dots of size order $1 \mathrm{~mm}$ was placed on the surface of each phantom, providing the reference point locations for motion capture. In this experiment, white dots were used to provide the background contrast against the clear silicone necessary for successful motion tracking.

Both phantoms were actuated vertically, whilst resting on a horizontal plate, at $100 \mathrm{~Hz}$ frequency, $0.5 \mathrm{~mm}$ amplitude. Simultaneous images of the phantoms were taken at $18^{\circ}$ offsets throughout the motion cycle by two cameras aimed at the phantom surface, giving a total of 20 frames per camera. Processing this motion using standard photogrammetry techniques resulted in a three dimensional description of the motion of each point across the equivalent of one steady-state motion cycle [11]. This tracked motion was then converted to an orthogonal set of amplitude and phase values using a least-squares optimization algorithm [10]. To allow motion comparison with FE simulated displacements, an interpolation between the physical reference point locations and the surface of the FE mesh was performed [12]. Figure 2 shows the processed raw motion data from both heterogeneous phantoms, along with the motion from the soft homogeneous phantom previously studied [10]. These surface motions constitute the complete input data set for elasticity reconstruction in this experiment.

\subsection{Finite Element (FE) Simulation}

A cylindrical FE mesh containing approximately 169,000 linear tetrahedral elements and 31,000 nodes was created using preprocessing software Gambit ${ }^{\mathrm{TM}}$. The bottom face of the cylinder was displacement constrained in the horizontal plane and actuated with harmonic boundary conditions matching the experimental conditions. All phantom configurations were simulated using the same mesh, with material properties assigned nodally. FE simulations 
were performed on a dual-processor workstation with 2 GB RAM using the MUMPS sparse matrix solution package [1].

Results from homogeneous phantoms using the DIET method have shown that the storage modulus value, $E^{\prime}$, for the soft and hard silicone are approximately $30 \mathrm{kPa}$ and $100 \mathrm{kPa}$ respectively, with notable sensitivity to the value of Poisson's Ratio [10]. Before proceeding with the heterogeneous cases, this prior study was extended to identify a best-fitting value for Poisson's Ratio, $\nu$. The resulting material properties for both the hard and soft silicone are shown in Table 1 . The best estimates of damping ratio, $\zeta$, and $\nu$, for the soft and hard silicone were fixed for all simulations at these values, allowing the study to focus on storage modulus values.

With material property estimates for the soft and hard silicone in hand, a range of $\mathrm{FE}$ simulations were performed on the stacked and concentric geometries. A two-parameter, shape-based approach was used, where the stiffness distribution of each phantom was described by independent parameters representing the interface position, $P$, and stiffness contrast, $C$, between the soft and hard silicone regions [9]. The interface position was defined for the stacked geometry as $P_{s}$, the ratio of the interface height to the total phantom height. For the concentric geometry, the interface position was defined $P_{c}$, the ratio of the inclusion diameter to the total phantom diameter. Measured values of $P$ for both geometries are shown in Figure 1.

Two fundamentally different approaches to estimating the phantom stiffness distributions were considered. These two methods are referred to as corroborative and contradictive methods. Both methods are referenced to a null stiffness distribution, representing the case where the phantom being tested is entirely comprised of soft material.

The corroborative method assumes that this soft material modulus is known, and seeks experimental evidence in the form of matching displacement data that confirms the presence of any hard region. If the experimental evidence corroborates this presence, a region of hard material can subsequently be added with an estimated interface position and hard stiffness described by $P$ and $C$. Conversely, the contradictive method assumes that the hard material modulus is known, and seeks to estimate a soft modulus and interface position supported by the experimental evidence. Should the experimental evidence refute any distribution including a high stiffness region, the null result of entirely soft stiffness can still be returned by setting the size of the hard region to zero, $P=0 \%$, and estimating the soft stiffness value alone using the parameter $C$.

Two stiffness contrast parameters, $C$, were used during the course of the study. For the corroborative method, where the soft silicone storage modulus, $E_{s}^{\prime}$, was assumed to be known, the focus was on optimizing the stiffness contrast parameter $C_{c o r}=E_{h}^{\prime} / E_{s}^{\prime}$, where $E_{h}^{\prime}$ represents the hard silicone storage modulus. For the contradictive method, where the hard modulus was assumed known, the contrast parameter was defined as $C_{c o n}=E_{s}^{\prime} / E_{h}^{\prime}$. The definition of these contrast parameters was consistent across both geometries.

The experimental evidence for or against a particular distribution, $\theta$, was calculated as a mean squared error, $\Omega$, across all surface reference points, 


$$
\Omega=\frac{\sum_{i=1}^{R}\left\|\Re\left(d_{i}\right)-\Re\left(f_{i}\right)\right\|}{3 R}+\frac{\sum_{i=1}^{R}\left\|\Im\left(d_{i}\right)-\Im\left(f_{i}\right)\right\|}{3 R},
$$

where $f$ is the interpolated FE displacement field based on $\theta, d$ is the experimentally measured motion data, and $R$ is the number of reference points, each comprising three orthogonal components of motion.

\section{Results}

\subsection{Corroborative Method}

To test the ability of the corroborative method to estimate a heterogeneous phantom stiffness distribution, a set of FE simulations were performed assuming the $E_{s}^{\prime}=28 \mathrm{kPa}$ soft silicone modulus value from Table 1 . For both geometries, the interface position parameter, $P$, was varied from $0-100 \%$, and the stiffness contrast parameter, $C_{c o r}$, was varied to generate a range of hard modulus values in the range $E_{h}^{\prime} \approx 80-200 \mathrm{kPa}$. The resulting values of the error metric $\Omega$ from the parameter sweep performed on each geometry are shown in Figure 3.

The estimated stiffness contrast value in Figure 3(a) indicates a hard silicone modulus of $E_{h}^{\prime}=116 \mathrm{kPa}$ for the stacked geometry. The estimated interface position for this geometry was within $3 \%$ (relative) of measured position of $47 \%$. The location of minimum error for the concentric model was along the edge of the parameter domain in Figure 3(b), with neither $P_{c}$ nor $E_{h}^{\prime}$ successfully estimated. In this case, the evidence suggests that the parameter combination leading to overall minimum motion error is not bounded by the range of parameters simulated.

The sensitivity of the two-parameter corroborative method to variations in the assumed soft modulus value was tested by modifying $E_{s}^{\prime}$ by $\pm 7.5 \%$ and $\pm 15 \%$ to $24 \mathrm{kPa}, 26 \mathrm{kPa}, 30 \mathrm{kPa}$ and $32 \mathrm{kPa}$, and repeating the range of simulations. Results from the sweeps at $\pm 15 \%$ are shown in Figure 4 , with full results from all sweeps shown in Table 2. The estimated value for $P_{s}$ for the stacked geometry varied by only $3 \%$ (absolute) across the stiffness range, with all estimates of $P_{s}$ within $10 \%$ (relative) of the measured value. Stiffness contrast values corresponding to minimum $\Omega$ indicated a hard storage modulus of $E_{h}^{\prime}=116-122 \mathrm{kPa}$ for the stacked model.

Results from the additional concentric model corroborative sweeps in Figures 4(c)-(d) had varied success. When $E_{s}^{\prime}=32 \mathrm{kPa}$, the error metric based approach again failed to identify the presence and location of the hard inclusion, with minimum motion error corresponding to an interface position of $P=15 \%$. However, in the case where $E_{s}^{\prime}=24 \mathrm{kPa}$, the interface position was estimated to within $20 \%$ (relative) of the measured value, with the hard modulus estimated at $E_{h}^{\prime}=106 \mathrm{kPa}$. 


\subsection{Contradictive Method}

Parameter estimation using a contradictive method was tested by analyzing parameter sweeps based on the hard silicone modulus value of $E_{h}^{\prime}=103 \mathrm{kPa}$ from Table 1. Stiffness contrast values, $C_{c o n}$, were varied to cover a soft silicone storage modulus range of approximately $E_{s}^{\prime}=10-50 \mathrm{kPa}$, and the interface position, $P$, was varied from $0-100 \%$. The resulting motion error from these parameter sweeps is shown in Figure 5 for both geometries considered.

Stacked and concentric motion error plots in Figure 5 indicate a soft silicone storage modulus of $E_{s}^{\prime}=24-25 \mathrm{kPa}$. The estimated interface position for the stacked geometry was an exact match to the measured value of $47 \%$, while the concentric geometry interface position was estimated as $35 \%$, which is within $20 \%$ (relative) of the measured value of $P_{c}=42 \%$ from Figure 1 . The minimum motion error metric values using the contradictive method were lower for both geometries than in the corroborative case, indicating a closer match to measured displacements using this approach.

The sensitivity of the contradictive method to variations in the assumed hard modulus value was tested by varying $E_{h}^{\prime}$ by $\pm 7.5 \%$ and $\pm 15 \%$, to $88 \mathrm{kPa}$, $95 \mathrm{kPa}, 111 \mathrm{kPa}$ and $118 \mathrm{kPa}$, and repeating all simulations. Figure 6 shows the result of the $\pm 15 \%$ parameter sweeps, with Table 3 reporting all results. Results are consistent with the $E_{h}^{\prime}=103 \mathrm{kPa}$ case in Figure 5, with all simulations indicating a soft modulus of $E_{s}^{\prime}=24-26 \mathrm{kPa}$. The interface position for the stacked geometry was estimated within $7 \%$ (relative) of the measured value in both cases, while the concentric interface position was estimated to within $20 \%$ (relative) of the measured value.

The best match to the experimental data for both geometries was obtained using the contradictive method with $E_{h}^{\prime}=118 \mathrm{kPa}$. Figure 7 is a comparison of the simulated motion at the best fit parameter values when $E_{h}^{\prime}=118 \mathrm{kPa}$, and the measured motions, for both stacked and concentric geometries. The motion error metric $\Omega$ for the stacked and concentric geometries in these cases was $0.141 \mathrm{~mm}$ and $0.125 \mathrm{~mm}$ respectively. These values represent an average orthogonal amplitude error per reference point of $\frac{\Omega}{2} \approx 0.06-0.07 \mathrm{~mm}$, which less than $20 \%$ of the average surface motion amplitude.

Inherent to the contradictive parameter estimation method is the assumption that if the null stiffness is represented by an entirely soft phantom, the correct stiffness distribution will be returned. To test this hypothesis, measured motion data previously obtained from the soft homogeneous silicone phantom was used as reference motion data, and compared to sweep displacements using the contradictive method, where $E_{h}^{\prime}=103 \mathrm{kPa}$. The resulting error domains for the stacked and concentric cases are shown in Figure 8. The estimated interface position value for both geometries is $P=0 \%$, corresponding to a homogenous phantom, where estimated $E_{s}^{\prime}$ values indicate a soft modulus of $27-28 \mathrm{kPa}$. 


\section{Discussion}

The two parameters describing the stiffness distribution of the stacked geometry were consistently estimated correctly using both corroborative and contradictive approaches. Results from the concentric case showed the stiffness distribution can be consistently estimated for this more challenging geometry only when adopting the contradictive approach. The error plots in Figures 3 and 4 indicate that a corroborative approach to estimation of stiffness distribution is sensitive to any error in the assumed value for $E_{s}^{\prime}$. This sensitivity is more significant in the concentric case, where assumed soft modulus values of $E_{s}^{\prime}=28 \mathrm{kPa}$ and $E_{s}^{\prime}=32 \mathrm{kPa}$ led to failed parameter estimation. Because the a priori silicone stiffness values were estimated based on phantoms created at a different time, this sensitivity to $E_{s}^{\prime}$ renders the corroborative method unsuitable in a clinical sense.

Figures 5 and 6 provide evidence that parameter estimates using a contradictive approach have very little sensitivity to variations in $E_{h}^{\prime}$, as expected from the relatively long shear wavelength in the harder material. Estimated values for soft modulus and interface position showed relative variation of less than $10 \%$ across both geometries over a $30 \%$ range in the assumed value of $E_{h}^{\prime}$, indicating a sensitivity of approximately $33 \%$, which is below the typical threshold for a robust result. All six instances of the contradictive estimates indicated a consistent soft modulus value of $24-25 \mathrm{kPa}$.

The estimated silicone stiffness values using both corroborative and contradictive estimates did not agree with the previously estimated homogeneous silicone modulus values. In comparison with $E^{\prime}=28 \mathrm{kPa}$ estimated from the homogeneous study [10], all contradictive estimates from the heterogeneous phantoms indicated a soft modulus value of $E_{s}^{\prime}=24-25 \mathrm{kPa}$, and the lowest motion error values using the corroborative method came when assuming $E_{s}^{\prime}=24 \mathrm{kPa}$. Similarly, the lowest error metric values when using the contradictive method came when $E_{h}^{\prime}=118 \mathrm{kPa}$, and all corroborative estimates indicated a value for $E_{h}^{\prime}$ above the previously estimated value of $E_{h}^{\prime}=103 \mathrm{kPa}$. The range of values estimated for silicone stiffness indicates that the modulus values for the soft and hard silicone varied between homogeneous and heterogeneous phantoms, most likely due to the different batches of silicone used when preparing these phantoms.

In general terms, the error metric for the stacked geometry had a higher sensitivity to variations in the interface position parameter than for the concentric case, as evidenced by highly ellipsoidal motion error contours, for example in Figure 3(a). This heightened sensitivity is expected as the stacked geometry has reference motion measurement located directly on the hard and soft silicone regions, allowing the interface position to be accurately identified due to the step change in shear wavelength across the two materials. The wavelength change between the lower and upper portions of the stacked phantom is visible in Figure 7(a). The concentric geometry had a significantly lower error sensitivity to interface position, and consequently less accurate interface position estimates. The physical separation of the measurement locations from the stiffness interface provides evidence as to why this loss of accuracy occurs. 
The success of the contradictive estimation approach when applied to the soft homogenous silicone phantom data shown in Figure 8, confirmed that this method was able to successfully return the null distribution when applied to a phantom with constant stiffness. The estimated value for the soft silicone modulus across both geometries of $27-28 \mathrm{kPa}$ matched the corresponding value previously estimated from the soft phantom [10], while the interface position value of $0 \%$ confirmed the absence of any hard inclusion in both cases.

The comparison between the best-fit displacements and the measured motion shown in Figure 7 indicates that the largest differences in amplitude occur close to the actuation plate, where amplitudes are large. It is possible that geometric non-linearity causes the displacements at this point to be under-estimated by FE simulation, an effect that could be minimized by reducing the actuation amplitude.

Though the shape based approach to property distribution estimation involved very simple geometry in this study, the underlying principles allow such a method to be extended to more clinically relevant scenarios. In such cases, an increase in the number of parameters would allow the identification of irregular shaped inclusions using a more sophisticated geometry description. Such an increase in parameters would require non-linear methods for parameter reconstruction, as the problem domain becomes too large for exhaustive exploration using FE simulation. An extension to the heterogeneous study where $E_{s}^{\prime}, E_{h}^{\prime}$, and $P$ are estimated independently may provide a more complete method for reconstructing the elasticity distribution of the concentric phantom, which returned less accurate interface position results in this experiment.

\section{Conclusion}

The heterogeneous surface error study performed demonstrated that the DIET method can estimate the elasticity distribution of heterogeneous phantoms using a simple two parameter, shape-based approach with limited surface motion data as input. Trials of both corroborative and contradictive techniques indicated that the contradictive method is more robust in the presence of error in any a priori stiffness assumptions. Adopting a contradictive approach to estimating elasticity distribution has the potential to reduce the number of parameters required to perform shape-based elasticity reconstructions. In such a method, the stiffness of any inclusion is fixed, as the exact stiffness used has very little effect on the observed surface motion. Such an approach represents a potentially new avenue to inclusion imaging in elastography, as it is also applicable to problems characterized by a larger number of parameters, allowing more complicated geometries to be reconstructed with less computational effort. 


\section{References}

1. Amestoy, P., Guermouche, A., L'Excellent, J.Y., Pralet, S., Alessandrini, G., Morassi, A., Rosset, E.: Hybrid scheduling for the parallel solution of linear systems. Parallel Computing 32, 136-156 (2006)

2. Barr, R.: Clinical applications of a real-time elastography technique in breast imaging. In: Proceedings of the 5th International Conference on the Ultrasonic Measurement and Imaging of Tissue Elasticity, p. 51 (2006)

3. Gupta, S.: A new breast cancer test. Time 168(25) (2006)

4. Krouskop, T., Wheeler, T., Kallel, F., Garra, B., Hall, T.: Elastic moduli of breast and prostate tissues under compression. Ultrasonic Imaging 20, 260-274 (1998)

5. Madelin, G., Baril, N., de Certaines, J., Franconi, J., Thiaudiere, E.: NMR characterization of mechanical waves. Annual Reports on NMR Spectroscopy 53, 203-244 (2004)

6. Muthupillai, R., Rossman, P.J., Lomas, D.J., Greenleaf, J.F., Riederer, S.J., Ehman, R.L.: Magnetic resonance elastography by direct visualization of propagating acoustic strain waves. Science 269, 1854-1857 (1995)

7. Ophir, J., Céspedes, E., Ponnekanti, H., Yazdi, Y., Li, X.: Elastography: a quantitative method for imaging the elasticity of biological tissues. Ultrasonic Imaging 13, 111-134 (1991)

8. Parker, K., Gao, L., Lerner, R., Levinson, S.: Techniques for elastic imaging: A review. IEEE Engineering in Medicine and Biology Magazine 15, 52-59 (1996)

9. Peters, A., Chase, J., Houten, E.V.: Digital Image-based Elasto-Tomography: Combinatorial and hybrid optimization algorithms for shape-based elastic property reconstruction. IEEE Transactions on Biomedical Engineering (in review)

10. Peters, A., Chase, J., Houten, E.V.: Digital Image-based Elasto-Tomography: Mechanical property estimation of silicone phantoms. Medical \& Biological Engineering \& Computation 46, 205-212 (2008)

11. Peters, A., Milsant, A., Rouzé, J., Ray, L., Chase, J., Houten, E.V.: Digital Image-based Elasto-Tomography: Proof of concept studies for surface-based mechanical property reconstruction. JSME International Journal 47, 1117$1123(2004)$

12. Peters, A., Uwe-Berger, H., Chase, J., Houten, E.V.: Digital Image-based Elasto-Tomography: Non-linear mechanical property reconstruction of homogenous gelatine phantoms. International Journal of Information and System Sciences 2, 512-521 (2006)

13. Peters, A., Wortmann, S., Elliott, R., Staiger, M., Chase, J., Houten, E.V. Digital Image-based Elasto-Tomography: First experiments in surface based mechanical property estimation of gelatine phantoms. JSME International Journal 48, 562-569 (2005)

14. Richards, M., Barbone, P., Wu, T., Kopans, D., Moore, R.: X-ray tomosynthesis elastography: A feasibility study. In: Proceedings of the 2nd International Conference on the Ultrasonic Measurement and Imaging of Tissue Elasticity, p. 90 (2003)

15. Rouvire, O., Yin, M., Dresner, M., Rossman, P., Burgart, L., Fidler, J., Ehman, R.: MR elastography of the liver: Preliminary results. Radiology 240, 440-448 (2006)

16. Samani, A., Zubovits, J., Plewes, D.: Elastic moduli of normal and pathological human breast tissues: An inversion technique based investigation of 169 samples. Physics in Medicine and Biology 52, 1565-1576 (2007)

17. Sinkus, R., Siegmann, K., Tanter, M., Xydeas, T., Fink, M.: MR elastography is capable of increasing the specificity of MR mammography - Influence of rheology on the diagnostic gain. In: Proceedings of the 5th International Conference on the Ultrasonic Measurement and Imaging of Tissue Elasticity, p. 111 (2006) 


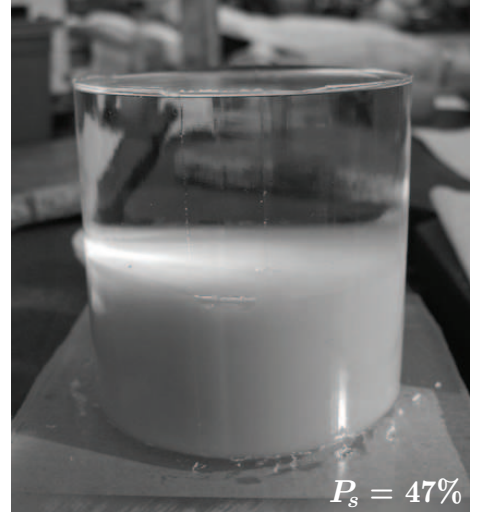

(a) Stacked phantom.

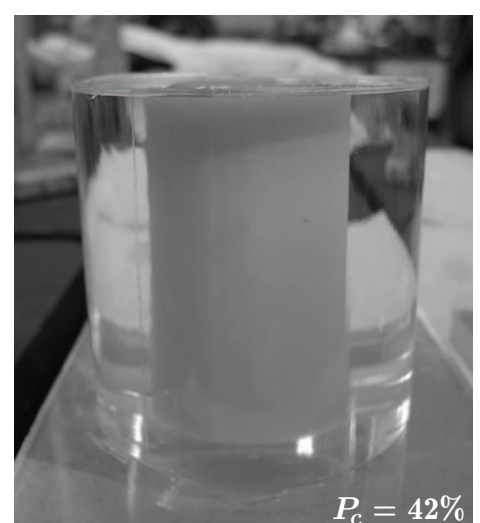

(b) Concentric phantom.

Fig. 1 The silicone phantoms following manufacture, where the interface between the hard silicone (opaque) and the soft silicone (clear) is visible, and the measured interface position between the hard and soft regions is indicated on each image. 


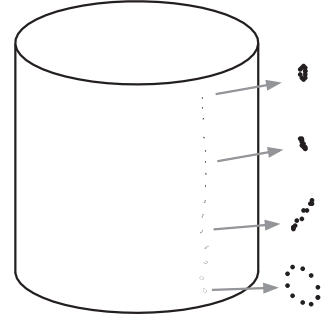

(a) Soft phantom.

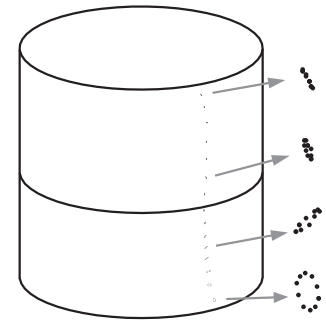

(b) Stacked phantom.

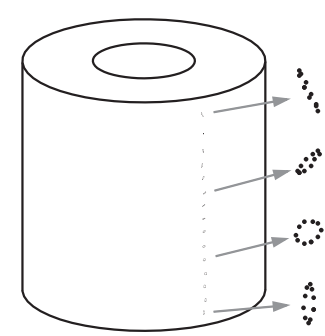

(c) Concentric phantom.

Fig. 2 The raw motion data captured by the camera system and processed into a fitted motion description, where four of the fifteen reference points are highlighted, and their motion shown in greater detail adjacent to the phantom. 


\begin{tabular}{lccc} 
& $E^{\prime}$ & $\zeta$ & $\nu$ \\
\hline Soft Silicone & $28 \mathrm{kPa}$ & 0.22 & 0.48 \\
Hard Silicone & $103 \mathrm{kPa}$ & 0.20 & 0.41 \\
\hline
\end{tabular}

Table 1 Summary of the silicone material properties estimated from homogenous phantom data using DIET. 


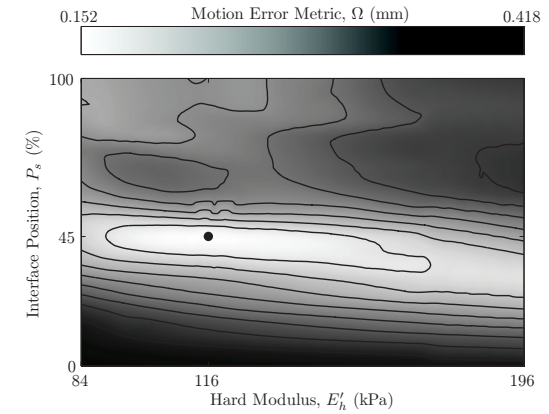

(a) Stacked phantom.

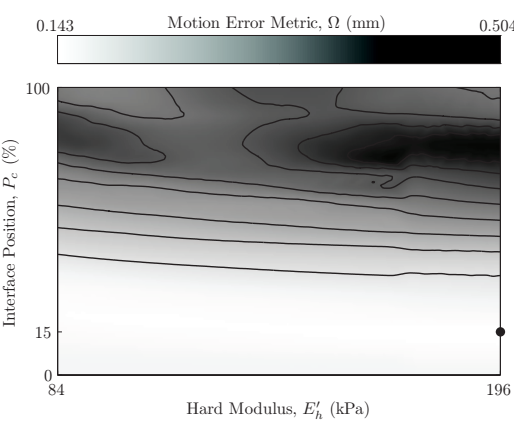

(b) Concentric phantom.

Fig. 3 Parameter sweep results for the heterogeneous phantoms, using the corroborative method with $E_{s}^{\prime}=28 \mathrm{kPa}$, where the location of minimum error on each plot is indicated $(\bullet)$. 


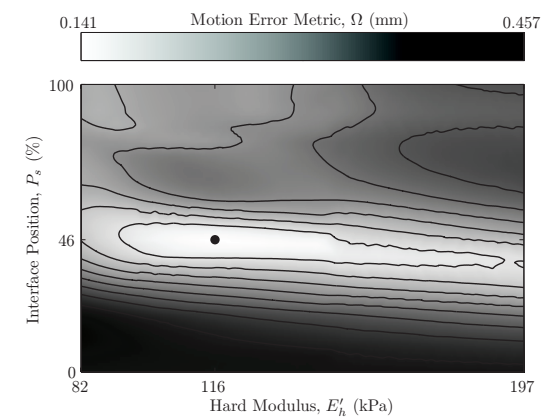

(a) Stacked phantom, $E_{s}^{\prime}=24 \mathrm{kPa}$.

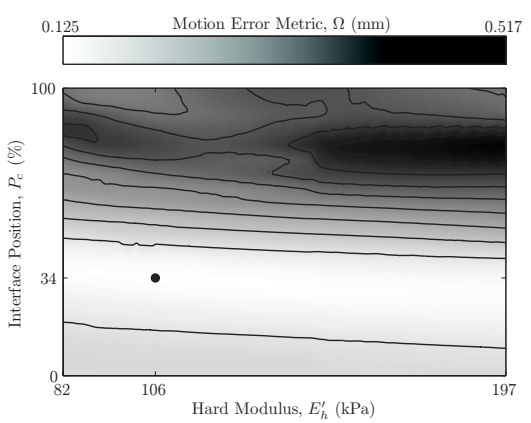

(c) Concentric phantom, $E_{s}^{\prime}=$ $24 \mathrm{kPa}$.

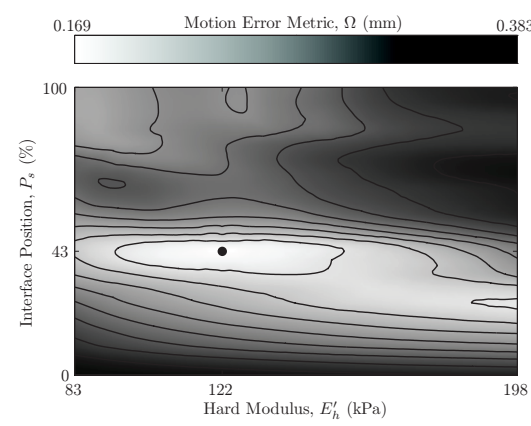

(b) Stacked phantom, $E_{s}^{\prime}=32 \mathrm{kPa}$.

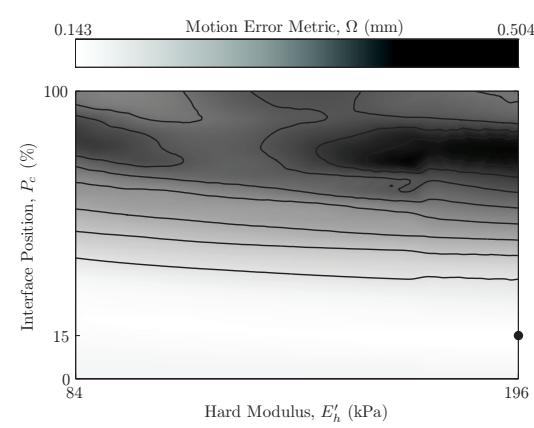

(d) Concentric phantom, $E_{s}^{\prime}=$ $32 \mathrm{kPa}$.

Fig. 4 Corroborative method sensitivity analysis results for the heterogeneous phantom data, where the location of minimum error on each plot is indicated $(\bullet)$. 


\begin{tabular}{llccccc} 
& & \multicolumn{6}{c}{ Assumed value for $E_{s}^{\prime}$} \\
Phantom & Value & $24 \mathrm{kPa}$ & $26 \mathrm{kPa}$ & $28 \mathrm{kPa}$ & $30 \mathrm{kPa}$ & $32 \mathrm{kPa}$ \\
\hline Stacked & $P_{s}(\mathrm{kPa})$ & 46 & 45 & 45 & 44 & 43 \\
& $E_{h}^{\prime}(\mathrm{kPa})$ & 116 & 118 & 116 & 118 & 122 \\
& $\Omega^{\prime}(\mathrm{mm})$ & 0.141 & 0.144 & 0.152 & 0.161 & 0.169 \\
Concentric & $P_{c}(\mathrm{kPa})$ & 34 & 25 & $15^{*}$ & $5^{*}$ & $15^{*}$ \\
& $E_{h}^{\prime}(\mathrm{kPa})$ & 106 & 130 & $196^{*}$ & $196^{*}$ & $196^{*}$ \\
& $\Omega^{\prime}(\mathrm{mm})$ & 0.125 & 0.132 & $0.143^{*}$ & $0.159^{*}$ & $0.143^{*}$ \\
\hline
\end{tabular}

Table 2 Summary of sweep sensitivity results using the corroborative stiffness estimation method. An asterisk indicates a result where the minimum error $(\Omega)$ occurred at the edge of the domain. 


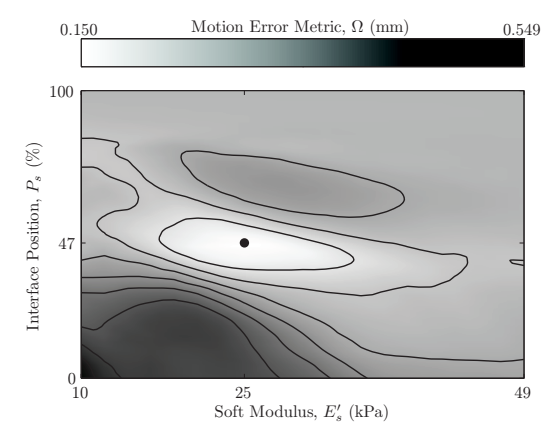

(a) Stacked phantom.

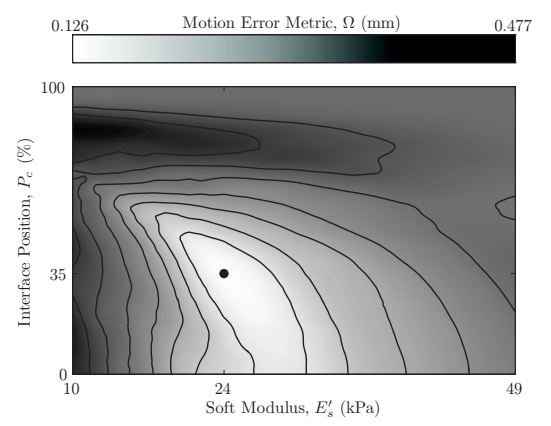

(b) Concentric phantom.

Fig. 5 Parameter sweep results for the heterogeneous phantom data, using the contradictive method with $E_{h}^{\prime}=103 \mathrm{kPa}$, where the location of minimum error on each plot is indicated $(\bullet)$. 


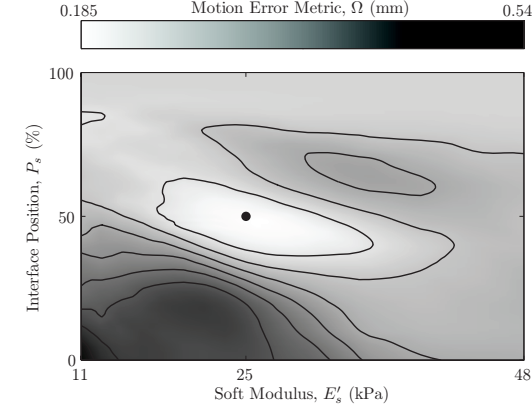

(a) Stacked phantom, $E_{h}^{\prime}=88 \mathrm{kPa}$

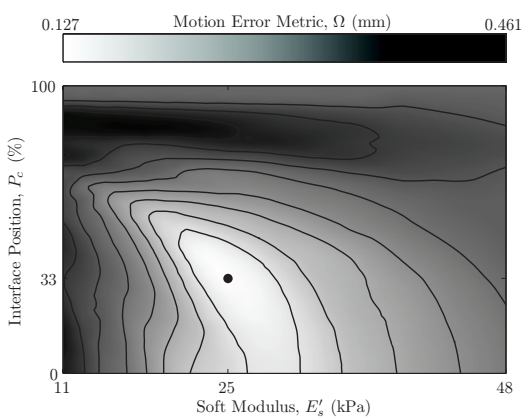

(c) Concentric phantom, $E_{h}^{\prime}=$ $88 \mathrm{kPa}$.

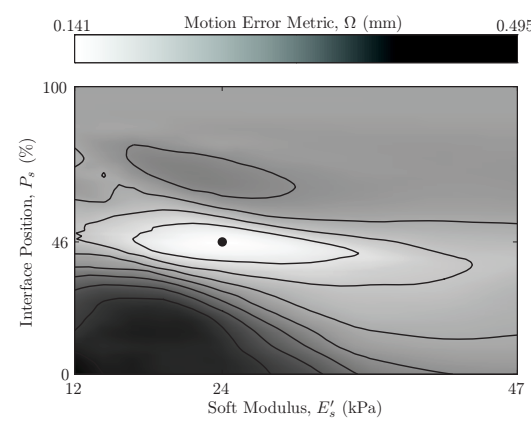

(b) Stacked phantom, $E_{h}^{\prime}=118 \mathrm{kPa}$.

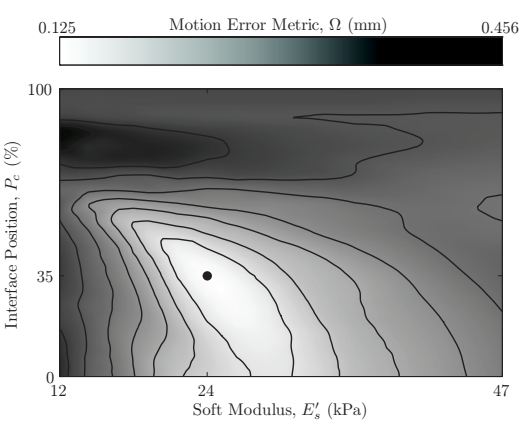

(d) Concentric phantom, $E_{h}^{\prime}=$ $118 \mathrm{kPa}$.

Fig. 6 Contradictive method sensitivity analysis results for the heterogeneous phantom data, where the location of minimum error on each plot is indicated $(\bullet)$. 


\begin{tabular}{llccccc} 
& & \multicolumn{5}{c}{ Assumed value for $E_{h}^{\prime}$} \\
Phantom & Value & $88 \mathrm{kPa}$ & $95 \mathrm{kPa}$ & $103 \mathrm{kPa}$ & $111 \mathrm{kPa}$ & $118 \mathrm{kPa}$ \\
\hline Stacked & $P_{s}(\mathrm{kPa})$ & 50 & 47 & 47 & 46 & 46 \\
& $E_{s}^{\prime}(\mathrm{kPa})$ & 25 & 26 & 25 & 24 & 24 \\
& $\Omega(\mathrm{mm})$ & 0.185 & 0.164 & 0.150 & 0.142 & 0.141 \\
Concentric & $P_{c}(\mathrm{kPa})$ & 33 & 35 & 35 & 35 & 35 \\
& $E_{s}^{\prime}(\mathrm{kPa})$ & 25 & 24 & 24 & 24 & 24 \\
& $\Omega(\mathrm{mm})$ & 0.127 & 0.127 & 0.126 & 0.127 & 0.125 \\
\hline
\end{tabular}

Table 3 Summary of sweep sensitivity results using the contradictive stiffness estimation method. 

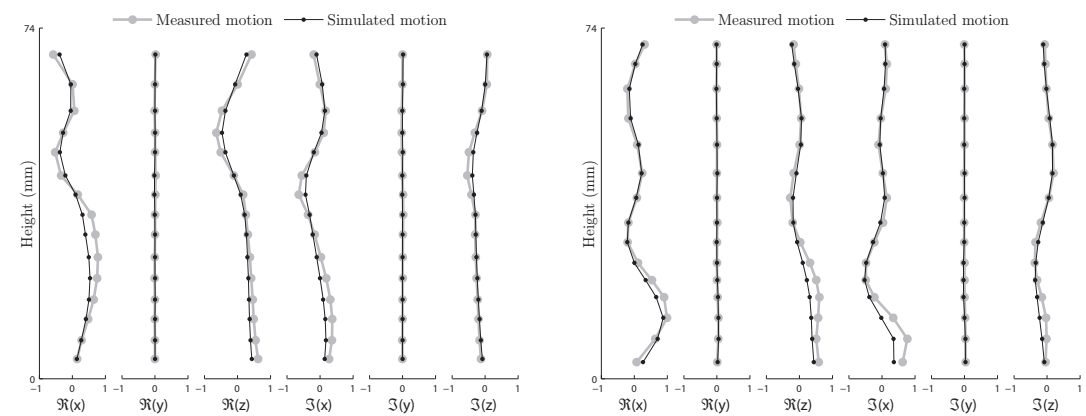

(a) Stacked phantom, simulated dis- (b) Concentric phantom, simulated displacement at $E_{h}^{\prime}=118 \mathrm{kPa}, E_{s}^{\prime}=$ placement at $E_{h}^{\prime}=118 \mathrm{kPa}, E_{s}^{\prime}=$ $24 \mathrm{kPa}, P_{s}=46 \%$. $24 \mathrm{kPa}, P_{c}=35 \%$.

Fig. 7 The best fitting FE-simulated cases compared with experimental motions for both heterogeneous silicone phantoms. 


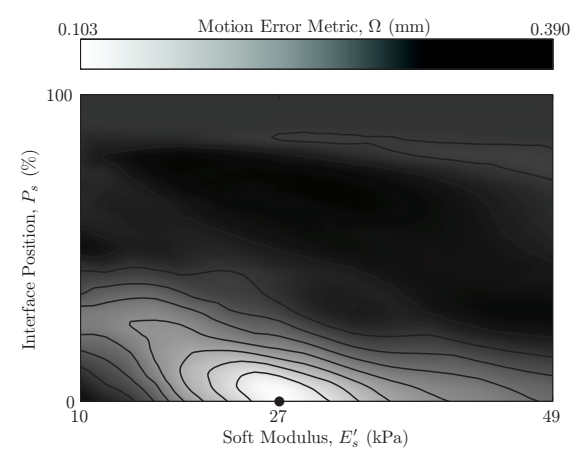

(a)

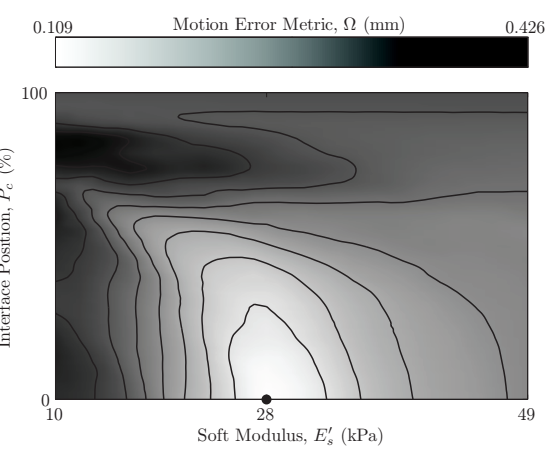

(b)

Fig. 8 Parameter sweep results from the homogenous soft silicone phantom, using the contradictive method with $E_{h}^{\prime}=103 \mathrm{kPa}$, where the location of minimum error on each plot is indicated $(\bullet)$. 